\title{
ELABORACIÓN DE CHICHA DE JORA A NIVEL DE PLANTA PILOTO, UTILIZANDO TECNOLOGÍA CERVECERA
}

Enrique de Florio R. $^{\prime}$

\author{
RE S U M EN
}

Se realizó el estudio de la elaboración de chicha de jora, incorporando operaciones de la tecnología cervecera, a nivel de planta piloto.

La elaboración del mosto por métodos cerveceros se inició con una relación de agua, sólidos de 1:4, cuya composición fue de $80 \%$ de jora y $20 \%$ de malta de cebada. El método de maceración fue mixto, se trató la jora como un cereal no malteado. El rendimiento del mosto fue de $63.58 \%$. La filtración se realizó con su propio "cake" y duró 30 minutos a una temperatura de $78^{\circ} \mathrm{C}$.

La fermentación dirigida en planta piloto con 1\% (del volumen del mosto) de levadura pastosa de $\mathbf{S}$. carlbergensis, con $60 \%$ de sólidos y una temperatura de $9^{\circ} \mathrm{C}$, duró aproximadamente 96 horas. A las 48 horas aparece el sabor agridulce, el que se va perdiendo hasta el final de la fermentación. La pasteurización cambió negativamente las características de la chicha.

\section{A B S T R A C T}

A study of the elaboration process of chicha de jora using beermaking technology was made at laboratory and pilot-plant level.

The obtention of must applying this beer-making tecnology started keeping an initial 1:4 water solid proportion implying this compositon of $80 \%$ jora and $20 \%$ barley malt. The macerating process

Was mixt treating thajora as a non-malted cerel, the must obtenined in percentage was made from the original cake at the temperature of 78 centigrade taking a time of 30 minutes.

the controlled fermentation al the pilot plant leve using $1 \%$ of must and using S. carlbergensis (as pasty yeast)mantaining a 60\% solid percentage and temperature of 9 centigrade lasted about 96 hours.

It was observed that 48 hours after the initiation of the process a bitter-sweet taste appers which tends to vanist at the end of the fermentation, also the final pasteurizing treatament changed the characteristies of the end product. 


\section{INTRODUCCIÓN}

La chicha de jora es una bebida alcohólica oriunda del Perú, cuyo origen se remonta a la época preincaica. Es ampliamente consumida en el Perú y en otros países latinoamericanos, constituyéndose en mercado potencial de consumo que posibilitaría su industrialización.

Actualmente, su elaboración es de carácter artesanal, observándose variaciones en el uso de insumos, métodos de elaboración y productos obtenidos. La elaboración tradicional es deficiente en cuanto a higiene y sanidad. Bajo estas condiciones la fermentación natural puede presentar características toxicológicas, por lo que existe una alta probabilidad de que el producto final no sea apto para el consumo humano (Bush, 1952).

El presente trabajo tiene como objeto establecer los parámetros para la elaboración de la chicha de jora a nivel de planta piloto, usando la tecnología cervecera.

\section{REVISIÓN BIBLIOGRÁFICA}

\subsection{DEFINICIÓN DE CHICHA DE JORA}

Se denomina chicha de jora, según Vásquez (1927), a la bebida alcóholica obtenida por fermentación, de la materia azucarada contenida en el maíz malteado.

\subsection{JORA O MALTA DE MAÍZ}

Se denomina jora al producto de la germinación controlada de los granos de maíz.

\subsection{ELABORACIÓN TRADICIONAL DE CHICHA DE JORA}

El proceso de elaboración de chicha de jora en términos más generales, presenta básicamente los siguientes pasos: jora, molienda, cocción, filtración, adición de azúcar y fermentación, como se muestra en la Figura №1 (De Florio,1986).

Manrique (1978) encontró al analizar 43 muestras diferentes de chicha de jora, que el $91 \%$ de éstas tenía el grado alcohólico entre $0.8 \%-5.7 \%$ en volumen, encontrándose hasta $13 \%$; en cuanto a la acidez se tuvo un $97 \%$ entre $0.48-3.97$, y $79 \%$ de las muestras un $\mathrm{pH}$ entre 3.5-4.6.

\subsection{PROCESOS DE LA TECNOLOGÍA CERVE-CERA APLICADOS A LA ELABORACIÓN DE CHICHA DE JORA EN LA PRESENTE INVESTIGACIÓN}

\subsubsection{Ma ceración}

\subsubsection{Objeto de la maceración}

1) Disolver sustancias de los ingredientes que sean solubles.
2) Solubilizar a través de la acción enzimática, las que sean insolubles en estado natural.

\section{FIGURA № 1: Flujo de operaciones en la elaboración de chicha de jora.}

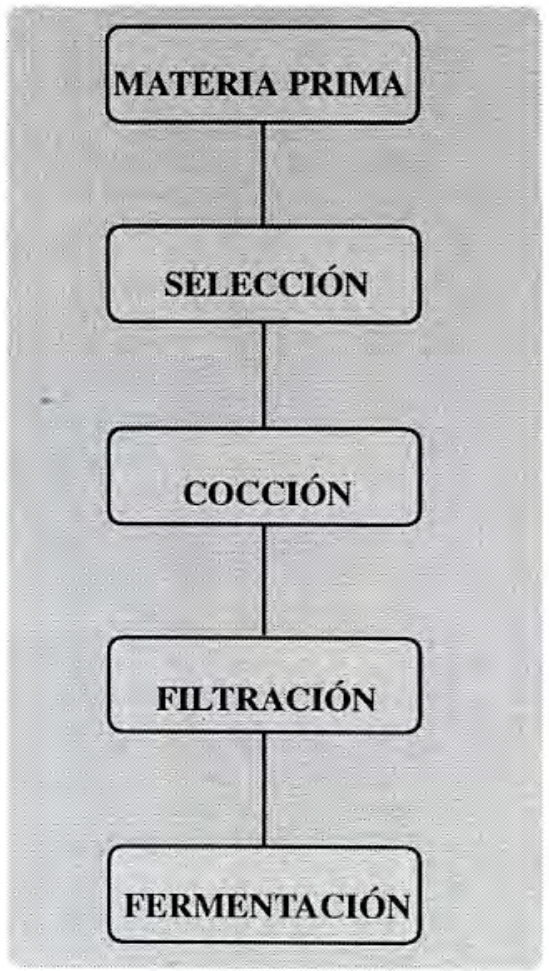

Fuente : Elaboración propia.

\subsubsection{Actividad de un producto malteado}

Consiste en la hidrólisis biológica de las materias amiláceas y proteicas que contiene, mediante enzimas producidas por el malteo (Carrasco,1954). En esta hidrólisis intervienen cuatro tipos de enzimas:

a. Amilolíticas: responsables de la solubilización de la fécula y su posterior sacarificación. Intervienen los siguientes grupos de enzimas:

a.1. Beta-amilasa: Actúa ordenadamente, produciendo maltosa sobre la cadena lineal de glucosa. Se obtiene $68-84 \%$ de maltosa, dependiendo del origen de la amilasa. La temperatura óptima de trabajo es de $60-70{ }^{\circ} \mathrm{C} \mathrm{pH}$, entre 4.6 - 5 (Carrasco,1954).

a.2. Alfa-amilasa: Actúa desordenadamente en enlaces 1,4 de almidón, produciendo unidades de dextrinas. La temperatura óptima de trabajo es 
de 70 - $76{ }^{\circ} \mathrm{C}$ y un $\mathrm{pH} 4.6-5$.

b. Proteolíticas: Desdoblan las proteínas en compuestos más sencillos, como péptidos, aminoácidos.

c. Fitasas: Enzimas importantes porque ayudan a establecer y mantener el $\mathrm{pH}$ de la mezcla agua-malta durante la maceración (ABMB, 1954).

d. Beta-gluconasas: Actúan sobre las beta-gluconasas que son un grupo lineal de polisacáridos, consistente en unidades de glucosa con enlaces $\mathrm{B}(1,4) 70 \%$, beta $(1,3) 30 \%$ que aumentan la viscocidad de la solución. Estos se encuentran en altas cantidades en maltas mal disgregadas y en cereales no malteados.

\subsubsection{Tratamiento de cereales no malteados}

Es imposible sacarificar el almidón de cereales no malteados, si no hay una mezcla directa del mismo con la malta. Por esta razón, deben estar previamente sujetos a una cocción.

Etapas: Gelatinización y sacarificación (conversión del almidón en compuestos más simples por acción de la enzimas). Insolubles al estado natural.

\subsubsection{Métodos de maceración}

- Infusión: La mezcla de sólidos no llega a ebullición.

- Decocción: Una parte del macerado es llevada a ebullición y retorna al tanque de maceración para elevar la temperatura del maceradó.

- Mixto: Combinación de los anteriores métodos. Dentro de este tipo, el más usado es el de doble alzada, donde existen mezclas individuales de adjuntos con $10-20 \%$ de malta molida.

\subsubsection{Filtración}

Se utiliza como medio filtrante su propio afrecho. Se requiere de un tiempo de reposo del mosto para la sedimentación de $15-30$ minutos y se realiza a $78^{\circ} \mathrm{C}$, realizando lavados continuos del lecho.

\subsubsection{Cocción}

Objeto de la maceración:esterilizar el mosto, destruir enzimas, precipitar proteínas que causan turbidez y concentración del mosto.

\subsubsection{Enfriamiento}

El objeto es la reducción de la temperatura de $100^{\circ} \mathrm{C}$ hasta la de siembra de $12^{\circ} \mathrm{C}$ y eliminar bacterias.

\subsubsection{Fermentación}

Un tiempo adecuado para el inicio de la fermentación se encuentra entre las 3 y 24 horas (A.B.M.A. 1977).

El ph óptimo para la fermentación es de 5 (A.B.M.A. 1977).

\subsubsection{Siembra}

La cantidad de levadura a usarse es de $0.5 \mathrm{lt} / \mathrm{hl}$ a 1 $\mathrm{lt} / \mathrm{hl}$ de levadura pastosa con $60 \%$ de sólidos.

\subsubsection{Aireación}

El aire se esteriliza mediante filtros de fibra de vidrio. La cantidad de aire disuelto en el mosto debe ser de 8ppm (A.B.M.A. 1977).

\subsubsection{Maduración}

La maduración permite la precipitación de materias turbias, amorfas, levaduras, desarrollo del sabor, da brillantez a la cerveza y previene la oxidación.

\subsubsection{Pasteurización}

En la mayoria de cervecerías se utiliza la temperatura de $60^{\circ} \mathrm{C}$ por un tiempo de 15 minutos, lo cual da resultados excelentes para la $\boldsymbol{S}$. carlbergenisis.

\section{III.MATERIALES Y MÉTODOS}

El presente trabajo se realizó en los laboratorios del T.A.P.A. y laboratorio Mariano Tabusso de la UNA La Molina y de la Cía. Backus \& Jhonston S.A.

\subsection{MATERIA PRIMA}

Se utilizó jora de maíz (Zea mays L.) de la variedad conocida como alazán, adquirida en el mercado. Malta de cebada y azúcar blanca granulada.

\subsection{EQUIPO}

\subsubsection{Planta piloto: La cual tiene molinos, balanza, pailas, fermentadores automáticos, cámara de maduración, filtros de fondo cribado, bombas centrífugas.}

Contaba con las siguientes instalaciones: Aire estéril, vapor, agua blanda caliente y fria, agua dura fría y caliente. 


\subsection{MATERIALES DE VIDRIO Y REACTIṾOS}

- Cloruro de sodio USP.

- Ácido clorhídrico 99\%

- Otros materiales y reactivos.

Se utilizó materiales de vidrio y reactivos adecuados para los análisis físicos, químicos y microbiológicos

\subsection{DISEÑO EXPERIMENTAL}

El diagrama del diseño experimental se encuentra en la Fig. 2, el cual consta de los siguientes puntos:

\subsubsection{Evaluación de parámetros de elaboración de chicha de jora por métodos de tecnología cervecera a nivel de laboratorio.}

\subsubsection{Evaluación de la calidad maltera de la chicha de jora.}

A la materia prima se le sometió a los siguientes análisis de humedad inicial, proteína total, proteína soluble, rendimiento en extracto, extracto original, poder diastásico, velocidad de filtración; asimismo se practicaron estos análisis sobre malta de cebada utlilizada en esta investigación.

3.4.1.2. Evaluación del mosto de chicha de jora, obtenido por el método de maceración mixto, sustituyendo cantidades porcentuales de jora por malta de cebada.

Se pesó muestras de $50 \mathrm{gr}$. de sólidos, en las que su composición se había sustituido porcentualmente jora por malta de cebada en un 10\%, 20\%, 30\%, 40\%, con respecto al peso total de los sólidos.

El método de maceración mixto se dio en las siguientes etapas:

a.Pre-cocción: Se agregó 176 cc de agua de cocimiento a los vasos de $600 \mathrm{cc}$ de capacidad, previamente tapados y codificados, llevándolos a "baño maría" a $52^{\circ} \mathrm{C}$. Cuando el agua de cocimiento estuvo en esta temperatura, se le agregó jora y en tanto que la malta se adicionó el $50 \%$ de la cantidad a utilizarse. Se elevó la temperatura del mosto, siguiendo la rutina de tiempos y temperaturas que se muestran en el Cuadro № 2 .

b. Maceración: Una vez terminada la pre-cocción, se enfrió el mosto a la temperatura de $52^{\circ} \mathrm{C}$ y se agregó el resto de la malta que se encuentra en un vaso de $200 \mathrm{ml}$. de agua de cocimiento, cuya temperatura inicial de esta mezcla es de $52^{\circ} \mathrm{C}$.
Se colocó la nueva mezcla en "baño maría" y se utilizó la rutina de tiempos y temperaturas para esta etapa que se encuentra descrita en el Cuadro № 2 . Una vez llegado al término de la maceración, se enfrió en "baño maría" y se llevó a un peso de 450 gr. con agua destilada. Se tomó el tiempo que dura la filtración para determinar su velocidad.

Sobre el filtrado se controló: $\mathrm{pH}$, extracto original, azúcares reductores, rendimiento en extracto, pruebas cualitativas de azúcares, examen organoléptico. En este último se determinó la partipación de la malta de cebada en la composición de la materia prima.

\section{FIGURA № 2 Diseño experimental de} elaboración de chicha de jora.

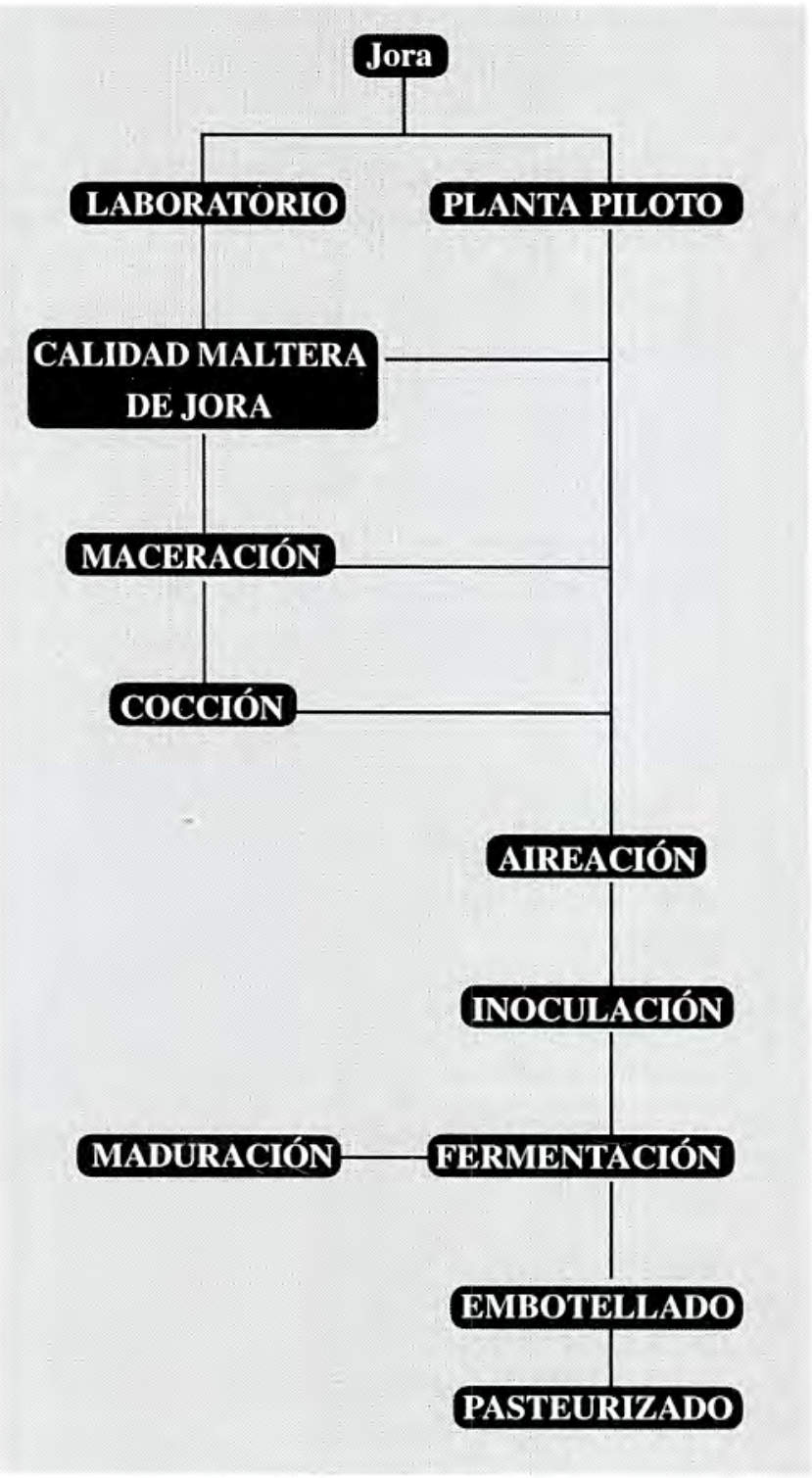


CUADRO № 2:Temperaturas y tiempos, utilizando el método de maceración mixto.

ETAPA DE PRE-COCCIÓN

\begin{tabular}{|c|c|}
\hline Temperatura ( ${ }^{\circ} \mathrm{C}$ ) & Tiempo (minutos) \\
\hline $50-52$ & 30 \\
$52-70$ & 20 (elevar) \\
$70-72$ & 30 \\
$70-100$ & 20 (elevar) \\
100 & 20 \\
\hline \multicolumn{2}{|c|}{} \\
ETAPA DE MACERACIÓN \\
\hline Temperatura ( ${ }^{\circ} \mathrm{C}$ ) & Tiempo (minutos) \\
\hline \multicolumn{3}{|c|}{} \\
$50-52$ & 30 \\
$52-70$ & 20 (elevar) \\
$70-72$ & 60 \\
$70-76$ & 10 (elevar) \\
76 & 15 \\
$76-78$ & 5 (elevar) \\
$78-80$ & 10 \\
\hline
\end{tabular}

3.4.1.3. Evaluación de la cocción del mosto de chicha de jora.

Se elaborará del mosto de jora, utilizando la formulación y el método empleado en.3.4.1.2.

La filtración utilizará como medio filtrante el mismo "cake" y se realizó a $78^{\circ} \mathrm{C}$.

El mosto se someterá a una cocción de 120 minutos y a los 15 minutos se le agregó $20 \mathrm{gr}$. de azúcar.

Se tomaron muestras a los 30, 60, 90 y 120 minutos de cocción y se le aplicó los siguientes ánalisis: Color, proteínas, turbidez, extracto fermentable (este último a los $120 \mathrm{~min}$.), pH, atenuación límite, estimación de la estabilidad biológica.

\subsubsection{Evaluación de la chicha de jora, elaborada en planta píloto, empleando la tecnología cervecera.}

En la Figura № 3 , se encuentra el diagrama de operaciones, el mismo que consiste en las siguientes etapas :

\section{a. Materia prima}

- Jora:se utlizará $10 \mathrm{~kg}$ de jora, cuyo tamaño de partícula clasificada como triturada dio un $78 \%$ de partículas entre las mallas № 18 y 10 . La jora fue el $80 \%$ de la composición de los sólidos al inicio de la elaboración del mosto.

- $2.5 \mathrm{Kg}$ de malta de cebada, cuya molienda sólo implicó un partido del grano (equivale al $20 \%$ de los sólidos al inicio de la cocción).

- $5 \mathrm{~kg}$ de azúcar blanca granulada (equivalente al $50 \%$ de la cantidad de la jora).

\section{b. Maceración}

Se utilizó el método de maceración mixta para elaborar el mosto, el cual consistió en las siguientes fases :

b.1. Pre-cocción: en la paila de pre-cocción se agregó 44 Its. de agua de cocimento, se elevó la temperatura a $52^{\circ} \mathrm{C}$ y al llegar a ésta, se añadió 1 $\mathrm{kg}$ de malta de cebada y luego los $10 \mathrm{~kg}$ de jora, dando una relación sólidos/agua de 1:4. Se elevó la temperatura de la paila, siguiendo las temperaturas y tiempos mostrados en el Cuadro № 2. Al final de la precocción, el mosto fue trasvasado a la paila de mezcla por acción de la gravedad. En el diagrama de la Fig.№3, se muestra la secuencia utilizada en el presente trabajo para elaborar la chicha de jora a nivel de planta piloto.

b.2. Maceración: En la paila de maceración se tiene en agitacion $1.5 \mathrm{~kg}$ de malta de cebada en $16 \mathrm{lt}$. de agua de cocimiento a una temperatura de $10^{\circ} \mathrm{C}$ en agitación, 10 minutos antes del ingreso del mosto proveniente de la paila de precoccíon. Una vez mezclados los mostos de las dos pailas, se bajó la temperatura a $52^{\circ} \mathrm{C}$, ayudados por el enfriamiento exterior de la paila. Se elevó la temperatura de la mezcla, utilizando la rutina que se muestra en el Cuadro № 8.

c. Filtración: Con ayuda de una bomba centrífuga, el mosto fue elevado al filtro donde reposa por espacio de 20 minutos para la formación del "cake" con el afrecho. El filtrado cayó dentro la paila de lúpulo, el afrecho se lava con agua de cocimiento a $78^{\circ} \mathrm{C}$.

d. Cocción: El mosto en la paila de lúpulo fue calentado, inyectando vapor y a los 5 minutos de iniciado el calentamiento se agregó el azúcar suavemente. Se elevó la temperatura del mosto para una agitación vigorosa.

La cocción se llevó a cabo por espacio de una hora. 
FIGURA № 3: Diagrama de flujo en la elaboración de chicha de jora en planta piloto.

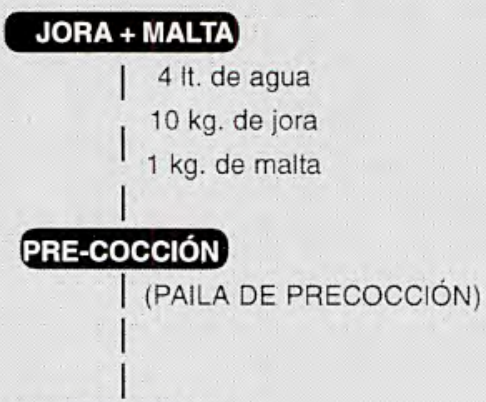

20 it. agua

\section{MACERACION}

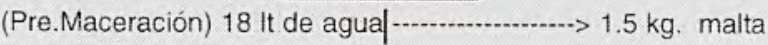

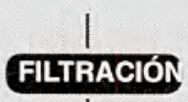

|.... Afrecho $3.969 \mathrm{~kg}$.
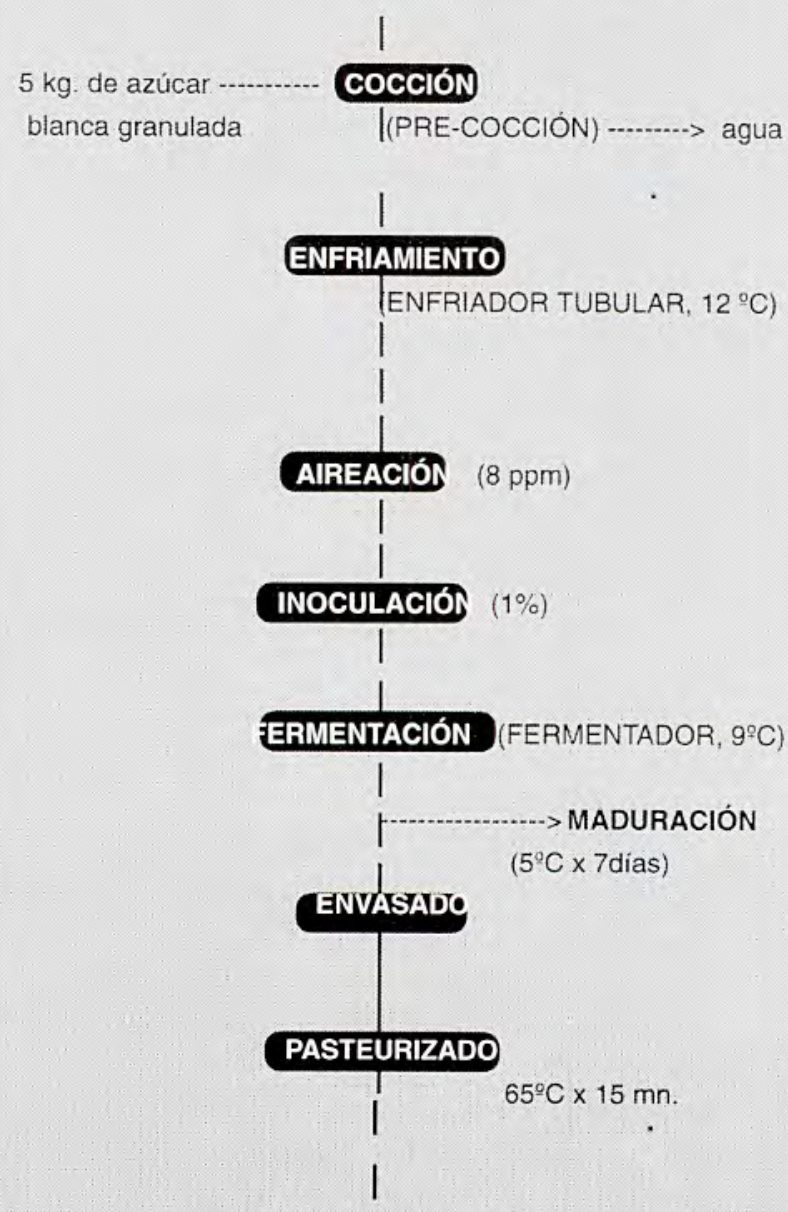

ALMACENADO e. Enfriamiento: El mosto descenderá de la paila de cocción por acción de la gravedad a través de un intercambiador de calor que lleva la temperatura del mosto a $12^{\circ} \mathrm{C}$.

Se tomará una muestra para controlar: atenuación límite, color, $\mathrm{pH}$, proteínas, azúcares reductores, acidez, extracto real, rendimiento del proceso, examen organoléptico.

f. Aereación del mosto: Se inyectará aire estéril al mosto, en un parámetro establecido de 8ppm.

g) Inoculación: Rápidamente se siembra la cantidad de $1 \%$ del volumen del mosto con una masa pastosa de $60 \%$ de sólidos de levadura S. carlsbergensis.

h. Fermentación: Se disminuirá la temperatura a $9^{\circ} \mathrm{C}$, mediante el sistema automático de control de temperatura de fermentación .

Se tomarán muestras cada 24 horas, donde se controlará : Temperatura del mosto, color extracto fermentable $\mathrm{pH}$, azúcares reductores, acidez, alcohol en volumen, extracto real, extracto aparente, examen organoléptico.

j. Embotellado y pasteurización: A las 78 horas de iniciada la fermentación, se procedió a embotellar la chicha de jora, se inyectó gas al fermentador y se llenaron botellas de $630 \mathrm{ml}$., las cuales se selló manualmente con ayuda de un taponador.

Las botellas de chicha embotellada se pasteurizarán a una temperatura de de $65^{\circ} \mathrm{C}$ por espacio de 15 minutos, luego se enfría a $10^{\circ} \mathrm{C}$.

A las muestras se les realizará los siguientes análisis: $\mathrm{pH}$, color, azúcares reductores, extracto aparente, examen organoléptico.

k. Maduración: Una vez concluida la fermentación, se trasvasará al tanque de maduración, a una temperatura de $5^{\circ} \mathrm{C}$ y se le evaluará por espacio de siete días.

\subsection{MÉTODOS DE ANÁLISIS}

\subsubsection{Métodos de análisis físico}

a. Determinación del $\mathrm{pH}$

Método potenciométrico, método oficial de A.S.B.C.(1957). 
b. Determinación de la gravedad específica.

Método hidrométrico, método oficial de A.S.B.C. (1975).

c. Determinación del extracto de harina fina o gruesa. Método gravimétrico, método oficial de la A.S.B.C. (1957).

d. Determinación del color (S.R.M.).

Método colorimétrico, método oficial de la A.S.B.C. (1957).

e. Determinación del extracto aparente.

Método gravimétrico, método oficial de A.S.B.C. (1957).

f. Determinación del alcohol de la cerveza.

Método por destilación, método oficial de la A.S.B.C. (1957).

g. Determinación del extracto real.

Método oficial de la A.S.B.C. (1957).

h. Determinación del grado alcohólico. Peso.

Método gravimétrico, método oficial de la A.S.B.C. (1957).

i. Determinación de la humedad.

Método oficial de la A.S.B.C. (1957).

j. Determinación del tamaño de partícula.

Método gravimétrico, método oficial de la A.S.B.C. (1957).

\subsubsection{Métodos de análisis químico}

a. Determinación de proteínas.

Método microkjeldal, método oficial de la A.S.B.C. (1957).

b. Determinación de azúcares totales.

Método volumétrico, método oficial de A.S.B.C. (1957).

c. Determinación de dextrinas.

Método volumétrico, método oficial de la A.S.B.C. (1957). d. Determinación de azúcares reductores.

Método volumétrico de Lane, método Oficial de A.S.B.C. (1957).

e. Determinación de la acidez.

Método por titulación, método Oficial de A.S.B.C. (1957).

f. Determinación cualitativa de almidones.

Método colorimétrico, método oficial de la A.S.B.C. (1957).

g. Determinación del poder diastásico.

Método volumétrico. Método oficial de la A.B.S.C (1957).

\subsubsection{Métodos de análisis microbiológico}

a. Determianción del extracto fermentesible. Método gravimétrico de la A.S.B.C (1957).

b. Determinación de la estabilidad bilológica.

Método del laboratorio de microbiología del laboratorio central de control de calidad de la Cía. Backus \& Jhonston (1978).

\subsubsection{Método de análisis organoléptico}

Se utilizó un panel entrenado para el análisis organoléptico.

\section{IV.RESULTADOS Y DISCUSIÓN}

\subsection{EVALUACIÓN DE LOS PARÁMETROS DE ELABORACIÓN DE CHICHA DE JORA POR MÉTODOS DE LA TECNOLOGIÁ CERVECERA A NIVEL DE LABORATORIO}

\subsubsection{Evaluación de la calidad maltera de jora de maíz alazán}

Los resultados se encuentran en el Cuadro № 3 , donde además se incluye el análisis de la malta de cebada para su comparación.

En el cuadro mencionado se observa que el poder diastásico de la jora es el $4.9 \%$ de la malta de cebada utilizada e indica que su capacidad de hidrolizar almidón es casi nula, debido quizá a la forma de malteo y/o 
conservación. Velásquez (1979) señala que un maíz malteado adecuadamente puede alcanzar hasta 121으.

Los resultados de los análisis del extracto indican que el elaborado con jora presenta almidón, posiblemente porque la jora carece de enzimas amilolíticas, pero de rendimiento más bajo. Las características organolépticas de cada uno de los extractos son muy diferenciadas.

Asimismo, la velocidad de filtración del mosto de jora es muy lenta, debido a la presencia de almidón y betaglucanos (restos de paredes celulares) que bloquean los poros del papel filtro, como señala la A.S.B.C. (1977).

\subsubsection{Evaluación del mosto obtenido por el método de maceración mixto, sustituyendo diferentes cantidades porcentuales de jora por malta de cebada}

Los resultados se encuentran en el Cuadro Nro 4, donde se observa un aumento de sustitución de malta por jora, incrementando el rendimiento en extracto, debido a la hidrólisis realizada por las enzimas de la malta.

Las características del mosto de jora mejoran: La velocidad de filtración es rápida, sólo hay presencia de almidón en la muestra de menor sustitución de jora por malta.

En el Cuadro No 3 se observa que el análisis organoléptico de extracto de jora indica que en cantidades superiores al $30 \%$ de sustutución se alteran significativamente las características típicas del mosto de jora. Se considera como ideal, la muestrả en la que se sustituye el $20 \%$ de jora por malta, porque la alteración es mínima y se mejora el rendimiento.

El pH es ideal para el incio de la fermentación. Asimismo, el porcentaje de azúcares se incrementa como consecuencia de la sustitución de jora por malta, debido a la presencia de enzimas amilolíticas.

\subsubsection{Evaluación de la cocción}

Los resultados se encuentran graficados en la Fig № 4, donde se observa que el color aumenta por una ligera caramelización de los azúcares por efecto del calor, como señala de Cleark (1957), al hablar de mostos cerveceros.

Las proteínas mantienen una ligera disminución por una precipitación provocada por del calor.

El valor de $\mathrm{pH}$ y el valor de la atenuación límite indicaron que el mosto tiene cualidades óptimas para fermentar.
La prueba de estabilidad biológica determinó que se obtienen resultados óptimos a partír de los 30 minutos de cocción.

\subsection{EVALUACIÓN DE LOS PARÁMETROS DE ELABORACIÓN DE CHICHA DE JORA POR MÉTODOS DE TECNOLOGIÁ CERVECERA A NIVEL DE PLANTA PILOTO}

La relación de agua sólidos al inicio del cocimiento fue $4: 1$, lo que permitió el flujo de la mezcla.

El rendimiento de la mezcla de sólidos es de $63.58 \%$, comparando este resultado con el rendimiento del mosto obtenido por métodos tradicionales en experiencias preliminares que fue de 37.95 , muestra una superioridad de $68 \%$ y este incremento se debe a la acción de las enzimas de la malta.

Los resultados se pueden observar en el Cuadro Nro. 5 y Fig. №5.

La atenuación límite indica que es una fermentación rápida y que el mosto es fácilmente fermentesible.

El color del mosto obtenido en planta piloto fue de 8.7오.R.M. y el mosto tradicional fue de 13.24 ㅇ.R.M., debiéndose a que el mosto de planta piloto tenia malta de cebada que disminuía el color.

Los resultados del análisis organoléptico muestran que las mejores características organolépticas se obtienen a las 48 horas de iniciada la fermentación.

$\mathrm{El} \mathrm{pH}$ de 4.48 del mosto de planta piloto fue apto para el inicio de la fermentación alcohólica.

Los cambios físico-químicos de la chicha de jora a través del tiempo de fermentación se encuentran en el

\section{CUADRO № 3: Comparación de la calidad maltera de la jora y la malta de cebada.}

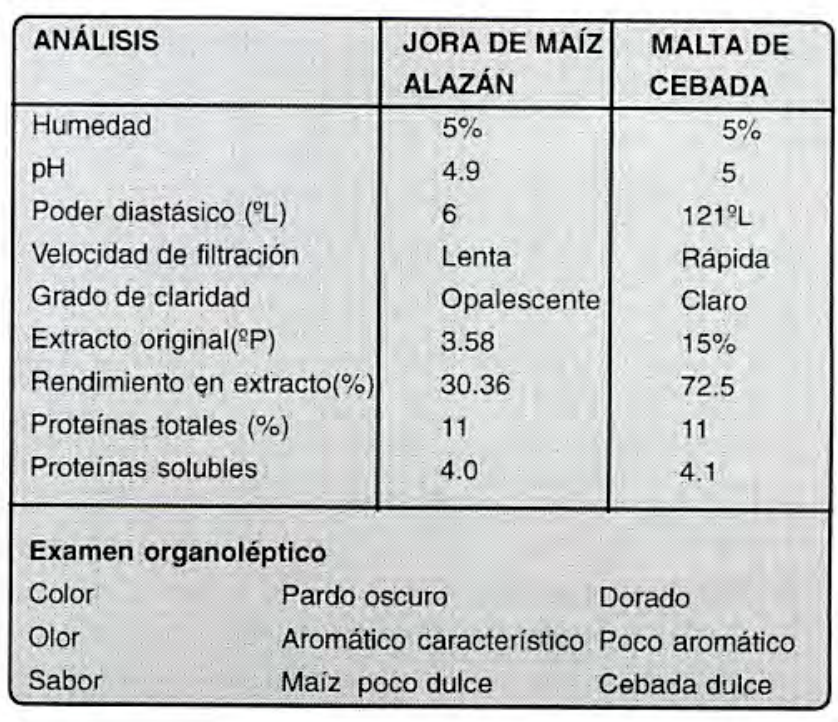


CUADRO № 4: Evaluación físico-química de los mostos de jora, elaborados por el método de maceración mixto, con sustitución de jora por malta de cebada.

\begin{tabular}{|l|c|c|c|c|}
\hline \multirow{2}{*}{ ANÁLISIS } & \multicolumn{3}{c|}{ CÓDIGO DE MUESTRAS } \\
\cline { 2 - 5 } & A & B & C & D \\
\hline Composición de sólidos (\%) & $90 \%$ jora/10\% malta & $90 \%$ jora/10\% malta & $70 \%$ jora/30\% malta & $60 \%$ jora/40\% malta \\
\hline $\mathrm{pH}$ & 5 & 5 & 5 & 5 \\
Extracto original (oP) & 6.7 & 7.3 & 7.8 & 8.82 \\
Azúcares reductores (\%) & 5.0662 & 5.14 & 5.46 & 5.82 \\
Rendiento en extracto (\%) & 58.44 & 63.144 & 68.85 & 72.69 \\
Prueba cualitativa & Amilodextrinas & Amilodextrinas & Amilodextrinas & Amilodextrinas \\
de azúcares y trazas de & & & & Rápida \\
almidón. & & Rápida & Rápida \\
Velocidad de & Normal & & & \\
filtración & & & & \\
\hline
\end{tabular}

Cuadro Nro 5 y la Figura № 5, donde se observa que la los mismos se realizan vigorosamente entre las 24 y 48 horas por la cantidad de extracto consumido y la producción se incrementa por acción del alcohol sobre los azúcares del mosto. A las 48 horas aparecen las caracteríticas organolépticas típicas, las que se van perdiendo hacia el final de la fermentación.

La pasteurización de la chicha de jora torna las características organolépticas negativas (sabor astringente) y el extracto aparente disminuye ligeramente por precipitación de la proteína. El color se parte de la chicha que no fue pasteurizada y llevada a la cámara de maduración a $5^{\circ} \mathrm{C}$, se encontró que $\mathrm{pH}$, color, acidez y extracto aparente, se mantienen constantes. El mosto se volvió brillante, de sabor no característico (negativo) y muy poco cuerpo.

\section{CONCLUSIONES}

1. El mosto elaborado con la tecnología cervecera, utilizando una dilución inicial de 4 partes de agua por una parte de sólidos, cuya composición era de $80 \%$ de jora triturada y $20 \%$ de malta, empleando el método de maceración mixto, elevó.los rendimientos de la jora en mosto en $68 \%$, comparándolo con los rendimientos obtenidos en la elaboración tradicional. Además se mejoró el tiempo de filtracíon que duraba 2 horas.

2. Con la levadura $\boldsymbol{S}$. carlbergensis se obtuvieron
Cuadro №5: Evaluación físico-química del proceso de fermentación de chicha de jora, elaborada en planta piloto, utilizando tecnología cervecera.

\begin{tabular}{|c|c|c|c|c|c|}
\hline \multirow[b]{2}{*}{ ANÁLISIS } & \multicolumn{5}{|c|}{ TIEMPO (horas) } \\
\hline & 0 & 24 & 48 & 72 & 96 \\
\hline Temperatura & 12 & 9 & 9 & 9 & 9 \\
\hline Atenuación limite & $4.45\left({ }^{\circ} \mathrm{P}\right)$ & - & - & - & - \\
\hline Color ('RM) & 8.7 & 8.7 & 8.7 & 8.7 & 8.7 \\
\hline $\begin{array}{l}\text { Extracto fermen- } \\
\text { tado }(\%)\end{array}$ & 0.0 & 31.95 & 65.38 & 69.73 & 69.96 \\
\hline $\mathrm{pH}$ & 4.48 & 4.42 & 4.1 & 4.0 & 4.0 \\
\hline Proteínas & 0.86 & ....... & - & - & 一. \\
\hline $\begin{array}{l}\text { Azúcares reduc- } \\
\text { tores }(\%)\end{array}$ & 8.64 & 5.25 & 2.11 & 1.94 & 1.9 \\
\hline $\begin{array}{l}\text { Acidez ( } \% \text { ácido } \\
\text { láctico) }\end{array}$ & 2.36 & 2.44 & 2.67 & 2.67 & 2.67 \\
\hline Alcohol ( $\%$ en vol.) & $\cdots$ & 4.0 & 5.5 & 7.0 & 7.0 \\
\hline Extracto real( $\left({ }^{\circ} \mathrm{P}\right)$ & 15.65 & 12.6 & 7.04 & 6.8 & 6.8 \\
\hline $\begin{array}{l}\text { Extracto aparen- } \\
\left.\text { te ( }{ }^{\circ} \mathrm{P}\right)\end{array}$ & $\cdots$ & 10.65 & 5.7 & 4.74 & 4.74 \\
\hline
\end{tabular}


características típicas de la chicha de jora.

3. El porcentaje de alcohol en volumen fue de $7 \%$. Se encuentra un tanto alto si lo comparamos con los resultados de Manrique(1978), asimismo, la conversión de azúcar en alcohol es alta.

4. La pasteurización no dio resultados satisfactorios para la conservación, porque altera las características organolépticas típicas de la chicha de jora .

5. Evaluación físico-química del proceso de fermentación de chicha de jora, elaborada en planta piloto, utilizando tecnología cervecera.

6. La maduración: la chicha de jora no ofreció cambios significativos en su composición físico-química, en tanto que las características organolépticas se tornan negativas.

7. La utilización de la tecnología cervecera a nivel de planta piloto, experimentada en este estudio, se adaptó apropiadamente a la elaboración de chicha de jora, excepto en lo que se refiere a la pasteurización y maduración.

\section{BIBLIOGRAFÍA}

1. American Society of Brewing Chemestry (1958). Methods of Analysis of Malt and Brewing.Wisconcyn, 6ta. ed.

2. Association of Brewing Master of America (1977). Practical Brewer. ABMA, 7ma. ed., págs. 92, 205-271.MadisonWisconcyn.

3. Bush. C. (1952). Consideraciones Médico-Sociales de la Chicha de Jora. Periódico Excelsior № 217, mayo-junio. Lima-Perú, páginas 25-27.

4. Carrasco T.J.(1954). Fabricación de Malta y Cerveza. Ed. Gráfica Rey Galdos,Madrid-España.

5. Milla, M. (1959). La Chicha. En Revista Cultural Peruana, vol XIX, pág 8 , Lima-Perú.
FIGURA № 5: Evaluación del proceso de fermentación dirigido en planta piloto

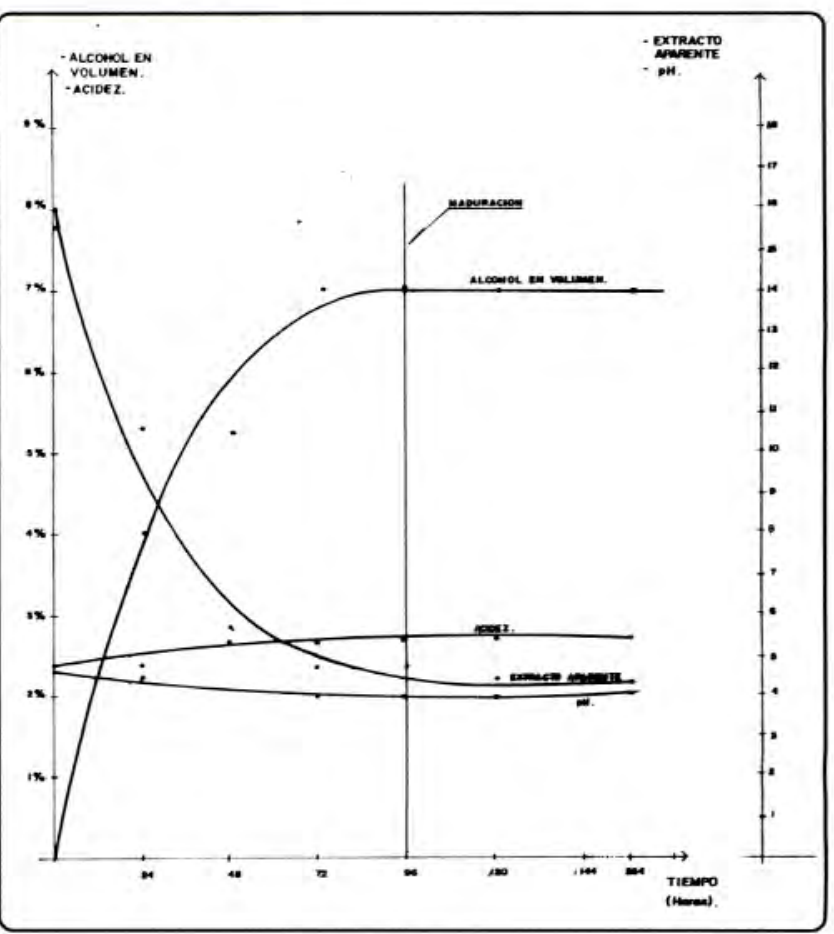

6. Manrique I. de Sáenz (1978). Flora Microbiana de la Chicha de Jora y Fermentación Experimental de la Levadura Seleccionada. Tesis de grado, programa de Farmacia de la UNMSM, Lima-Perú.

7. Muelle J. (1945). La Chicha en el Distrito de San Sebastián (Cusco). Revista del Museo Nacional de Cultura, tomo XIV, 1945, págs. 142-144, Lima-Perú.

8. Velásquez, M. (1979). Determinación de Parámetros para la Elaboración de Jora a partír de Maíz Cancha de Huaraz (Variedades terciopelo y rojo huarosanta).

9. Viñas, Eduardo (1956). La Composición Química de las Diferentes Chichas que se Consumen en el Perú. Ministerio de Salud Pública y Asistencia Social, departamento de Nutrición. Servicio Cooperativo Internacional de Salud Pública, págs. 1-3. Lima-Perú. 